\title{
Bladder Adenocarcinoma: About 7 Cases
}

\author{
I Adrif, S Daoudi, S Ouguellit, S Naciri, H Mrabti, H Errihani
}

\begin{abstract}
This is a retrospective study of 7 cases of primary adenocarcinoma of the urinary bladder collected during a period of 10 years (between 2007 and 2017) at the national institute of oncology of Rabat.
\end{abstract}

The aim of our study is to analyze the epidemiological, clinical, radiological, pathological, and treatment and prognosis of primary adenocarcinoma of the bladder.

The mean age of our patients was 51.5 years. The main risk factor found is tobacco $(57.1 \%)$. The macroscopic hematuria was the master symptom in all patients. The anatomopathological study revealed 4 histological types: 1 enteric adenocarcinoma, and 2 cases for each the signet ring cell, mucinous and not otherwise specified adenocarcinomas.

Two patients were metastatic at the time of diagnosis but they did not receive chemotherapy because of their general condition. For the five non-metastatic patients; two were lost to follow-up and three underwent surgical treatment by cystectomy. Two of the operated patients received adjuvant therapy.

During the follow-up, the 3 patients relapsed after an average delay of 18.3 months. The three patients received palliative chemotherapy with FOLFOX as the first line regiment for 2 cases and Gem CDDP for 1 case, but the three progressed. Two of them received a second line chemotherapy; FOLFIRI or Gem CDDP type. The patient who received FOLFIRI relapsed 16 months after the end of chemotherapy. Overall survival was $42.8 \%$ at 2 years versus $28.5 \%$ at 3 years.

Index Terms-Adenocarcinoma, Bladder, Chemotherapy, Pronosis.

\section{INTRODUCTION}

Primary adenocarcinoma of the urinary bladder is an exceptional tumor that represents 0.5 to $2 \%$ of all bladder malignancies.

We report the experience of the medical oncology service of the National Institute of Oncology in Rabat.

\section{MATERIEL AND METHODS}

This is a retrospective study of 7 cases of primary adenocarcinoma of the urinary bladder collected during a period of 10 years (between 2007 and 2017) at the national institute of oncology of Rabat.

ADRIF Imane, Department Of Medical Oncology, National Institute Of Oncology, Rabat, Morocco.

DAOUDI Sara, Department Of Medical Oncology, National Institute Of Oncology, Rabat, Morocco.

OUGUELLIT Sihame, Department Of Medical Oncology, National Institute Of Oncology, Rabat, Morocco.
Our analysis was based on the evaluation of the epidemiological, clinical, anatomopathological, therapeutic and prognostic characteristics of bladder adenocarcinoma.

\section{RESULTS}

The mean age of our patients was 51.5 years with extremes between 41 and 67 years. The male sex represents $85 \%$ of the cases ( 6 patients) with a sex ratio $=6$ ( 6 men for a woman). The main risk factor found is tobacco $(57.1 \%)$. The macroscopic hematuria was the master symptom in all patients. The histological diagnosis was made by endoscopic resection in all cases, and the anatomopathological study revealed 4 histological types: 1 enteric adenocarcinoma, and 2 cases for each the signet ring cell, mucinous and not otherwise specified adenocarcinomas.

Two patients were metastatic at the time of diagnosis and the metastatic site was pulmonary in one patient and bony in the other, but they did not receive chemotherapy because of their general condition. For the five non-metastatic patients; two were lost to follow-up and three underwent surgical treatment by cystectomy (radical in 2 cases and partial in one). Two of the operated patients received adjuvant therapy; the first was treated with 4 cures of Gem CDDP and the second by radiochemotherapy.

During the follow-up, the 3 patients relapsed after an average delay of 18.3 months (with extremes between 10 and 33 months). One patient had pulmonary metastatic relapse and the 2 others had a very locally advanced pelvic no operable relapse.

The three patients received palliative chemotherapy with FOLFOX as the first line regiment for 2 cases and Gem CDDP for 1 case, but the three progressed. Two of them received a second line chemotherapy; FOLFIRI or Gem CDDP type. The patient who received FOLFIRI relapsed 16 months after the end of chemotherapy. Overall survival was $42.8 \%$ at 2 years versus $28.5 \%$ at 3 years.

\section{DISCUSSION}

Primary bladder adenocarcinoma is the third most common epithelial tumor, accounting for $0.5-2 \%$ of all bladder tumors. It most commonly occurs in men with a sex ratio of 3 men to a woman, between 40 and 60 years of age $[1,2]$.

Owing to the rarity of the disease, the pathogenesis and natural history of primary bladder adenocarcinoma have not been well characterized [3].Several risk factors have been described, most notably, almost $90 \%$ of bladder tumors in 
patients with exstrophy of bladder are adenocarcinoma and up to $10 \%$ of all bladder cancers are adenocarcinomas in areas where schistosomiasis is endemic [3], [4]. Other possible risk factors include chronic irritation, obstruction, cystocele and endometriosis [4]. Cystitis glandularis and intestinal metaplasia are often found adjacent to bladder adenocarcinoma, but recent studies have showed that cystitis glandularis and intestinal metaplasia are not associated with an increased risk for adenocarcinoma [4].

The symptoms of adenocarcinoma do not differ from other kinds of bladder tumor. In our series, hematuria was the most frequent presenting symptom.

Abdominopelvic CT is essential to assess the locoregional tumor extension and determine the metastatic extension [5]. In practice, when the diagnosis of adenocarcinoma is made, the clinical problem is to eliminate a secondary tumor, metastasis or invasion of the bladder wall by a neighboring tumor (colon, prostate, endometrium or ovary) [4].

Microscopically, bladder adenocarcinoma is subdivided into enteric type, mucinous type, mixed type and not otherwise specified (NOS) adenocarcinoma. Additional morphological patterns have been described, such as hepatoid growth. Formerly, clear cell adenocarcinoma has been included as a variant of bladder adenocarcinoma, but is now considered as a separate disease entity within tumours of Mullerian type in the latest World Health Organisation (WHO) classification [6].

Therapeutically there is no standard treatment; these tumors are often treated by extrapolation to urothelial carcinoma and adenocarcinomas from other sites [7]. In the absence of an extra extension bladder treatment is based on excision surgery (total cystoprostatectomy in men or anterior pelvectomy in women), combined with extensive dissection [5].

The role of chemotherapy and radiotherapy in unresectable or metastatic disease is unclear, and no randomized trials have been completed [7].In a prospective study of ifosfamide, paclitaxel and cisplatin in 20 patients with non urothelial bladder cancer (11 of whom had adenocarcinoma), the response rate was $36 \%$ and the median survival was 25 months [7]. The same response rate was reported in a retrospective analysis of 21 patients who received first-line regimens containing cisplatin, including 14 with adenocarcinoma [7]. Other agents have been reported in case reports, such as 5-FU alone, 5-FU with doxorubicin and mitomycin C, FOLFOX and bevacizumab, with varying responses [7].

The prognosis for primary adenocarcinoma remains very poor; five-year survival, all stages combined, ranges from 0 to $31 \%$ [5].

\section{CONCLUSION}

Adenocarcinoma of the bladder represents a diagnostic and therapeutic challenge due to the scarcity of studies on this type of tumor, in most cases being small retrospective series. Until now, no codified therapeutic strategy has been established, but its treatment remains essentially surgical. The prognosis remains reserved, however, due to the very often aggressive and infiltrative nature of this histological sub-type.

\section{REFERENCES}

[1] S. Mellas et al. Mise au point sur les tumeurs non urothéliales de la vessie. J. Afr. Cancer (2011).

[2] G.Vasudevan and al. Bladder Adenocarcinoma: A Persisting Diagnostic Dilemma. Journal of Clinical and Diagnostic Research. 2017 Mar, Vol-11B.

[3] R.Dutta and al. Effect of tumor location on survival in urinary bladder adenocarcinoma: A population-based analysis urologic oncology 2016

[4] V.Dadhania and al. Adenocarcinoma of the urinary bladder Am J Clin Exp Urol 2015.

[5] F. Audenet and al. Adénocarcinome primitif de la vessie. Tumeurs rares en urologie : rein et vessie Vol. V - no 32014.

[6] Sanghui Park and al. Non-urothelial carcinomas of the bladder. (2019) Histopathology 74, 97-111.

[7] G. Tsironis and al.Treating bladder adenocarcinoma Transl Androl Urol 2018 